\title{
ON THE HOMOLOGY OF DOUBLE BRANCHED COVERS
}

\author{
RONNIE LEE AND STEVEN H. WEINTRAUB
}

(Communicated by Thomas Goodwillie)

Dedicated to Frank Raymond on the occasion of his sixtieth birthday

ABSTRACT. If $\pi: \tilde{X} \rightarrow X$ is a double branched cover, with branching set $F$, we relate $H_{*}\left(\tilde{X}: \mathbb{Z}_{2}\right), H_{*}\left(X: \mathbb{Z}_{2}\right), H_{*}\left(X, F: \mathbb{Z}_{2}\right)$, and $H_{*}\left(F: \mathbb{Z}_{2}\right)$.

In this note we present a pair of observations on the homology of double branched covers. These observations arose in our investigations [LW1, LW2], but because of the specialized nature of those investigations and the potential general utility of these observations, we have chosen to present them separately here.

All (co)homology in this paper is to be taken with $\mathbb{Z}_{2}$ coefficients.

Our first result is a simple generalization of the Gysin sequence, which, however, we have not been able to find in the literature.

Theorem 1. Let $\pi: \tilde{X} \rightarrow X$ be a twofold cover of the simplicial complex $X$, branched over a subcomplex $F$ of $X$. Let $A$ be an arbitrary subcomplex of $X$, and set $\tilde{A}=\pi^{-1}(A)$. If $T_{*}$ denotes the transfer map on homology; then there is a long exact sequence

$$
\cdots \rightarrow H_{i}(X, F \cup A) \stackrel{T_{*}}{\longrightarrow} H_{i}(\tilde{X}, \widetilde{A}) \stackrel{\pi_{*}}{\longrightarrow} H_{i}(X, A) \stackrel{d_{*}}{\longrightarrow} H_{i-1}(X, F \cup A) \rightarrow \cdots .
$$

Proof. If $T: C_{*}(X, A) \rightarrow C_{*}(\tilde{X}, \tilde{A})$ is the transfer map on chains, then there is a short exact sequence of chain complexes

$$
0 \rightarrow C_{*}(X, F \cup A) \stackrel{T}{\rightarrow} C_{*}(\tilde{X}, \tilde{A}) \stackrel{\pi}{\rightarrow} C_{*}(X, A) \rightarrow 0 .
$$

We suppose throughout the remainder of this paper that we are in the following situation (where we use the term "manifold" to mean connected, compact manifold):

Situation $(*), X$ is a smooth, and hence piecewise linear, $n$-manifold (possibly with boundary), and $\pi: \tilde{X} \rightarrow X$ is a double cover branched over a subcomplex $F$, where $F$ is the union (not necessarily disjoint) of codimension 2 smooth

Received by the editors September 8, 1992 and, in revised form, June 30, 1993.

1991 Mathematics Subject Classification. Primary 57M12.

The first author was partially supported by the National Science Foundation.

The second author was partially supported by the Louisiana Educational Quality Support Fund and the Sonderforschungsbereich für Geometrie und Analysis, Universität Göttingen. 
submanifolds $F_{1}, \ldots, F_{k}$ which are pairwise mutually transverse. Also, $F$ is transverse to $\partial X$, and hence $F \cap \partial X=\partial F$, which may be empty.

In Situation $(*)$, Theorem 1 yields the long exact sequence

$$
\begin{aligned}
\cdots \rightarrow & H_{i}(X, F \cup \partial X) \stackrel{T_{*}}{\rightarrow} H_{i}(\tilde{X}, \partial \tilde{X}) \\
& \stackrel{\pi_{*}}{\longrightarrow} H_{i}(X, \partial X) \stackrel{d_{*}}{\longrightarrow} H_{i-1}(X, F \cup \partial X) \rightarrow \cdots .
\end{aligned}
$$

Theorem 2. In Situation $(*)$, let $d_{*}$ be as in Theorem 1 and let $\partial_{*}$ be the composition of the boundary map of the triple $(X, F \cup \partial X, \partial F)$ with the projection of

$$
H_{*}(F \cup \partial X, \partial F) \cong H_{*}(F, \partial F) \oplus H_{*}(\partial X, \partial F)
$$

onto its first summand. Then the composite

$$
H_{n}(X, \partial X) \stackrel{d_{*}}{\rightarrow} H_{n-1}(X, F \cup \partial X) \stackrel{\partial_{*}}{\rightarrow} H_{n-2}(F, \partial F)
$$

satisfies

$$
\partial_{*} d_{*}([X, \partial X])=\left[F_{1}, \partial F_{1}\right]+\cdots+\left[F_{k}, \partial F_{k}\right] .
$$

Here $[X, \partial X]$ and $\left[F_{i}, \partial F_{i}\right]$ denote the fundamental homology classes of $(X, \partial X)$ and $\left(F_{i}, \partial F_{i}\right)$, and the sum lies in $H_{n-2}\left(F_{1}, \partial F_{1}\right) \oplus \cdots \oplus H_{n-2}\left(F_{k}, \partial F_{k}\right)$ $\cong H_{n-2}(F, \partial F)$.

Proof. First we consider the case $(X, \partial X)=(D, S)$, where $D$ is a 2-disk, $S$ is its boundary circle, and $F=C$, the center of $D$. (Then we may identify $\pi:(\tilde{X}, \partial \tilde{X}) \rightarrow(X, \partial X)$ with the map $z \mapsto z^{2}, z \in \mathbb{C},|z| \leq 1$.) Let $R$ be a radius of $D$. Then it is easy to check directly that $d_{*}([D, S])=[R] \in$ $H_{1}(X, F \cup \partial X)$ and $\partial_{*}([R])=[C] \in H_{0}(F)$ as claimed.

The exact sequence of Theorem 1 may be dualized to cohomology, and so we have a map $d^{*}: H^{i-1}(X, F \cup \partial X) \rightarrow H^{i}(X, \partial X)$. Then, if \{\} denotes the fundamental cohomology class, we have in the above case

$$
\delta^{*}(\{C\})=\{R\}, \quad d^{*}(\{R\})=\{D, S\} .
$$

Now for the general case. Consider a submanifold $F_{i}$ in $F$, and let $E_{i}$ be the complement of an open regular neighborhood of $F_{i} \cap\left(F_{1} \cup \cdots \cup F_{i-1} \cup F_{i+1} \cup\right.$ $\left.\cdots \cup F_{k}\right)$ in $F_{i}$. Let $P_{i}$ be a closed regular neighborhood of $F_{i}$ in $X$. Then we may identify $P_{i}$ with the total space of a $D^{2}$-bundle over $F_{i}$, and we assume that $P_{i}$ is chosen small enough so that if $N_{i}$ is the restriction of $P_{i}$ to $E_{i}$, then $N_{i} \cap F=E_{i}$. Let $T_{i}=\partial P_{i} \cap N_{i}$, an $S^{1}$-bundle over $E_{i}$. Consider the diagram

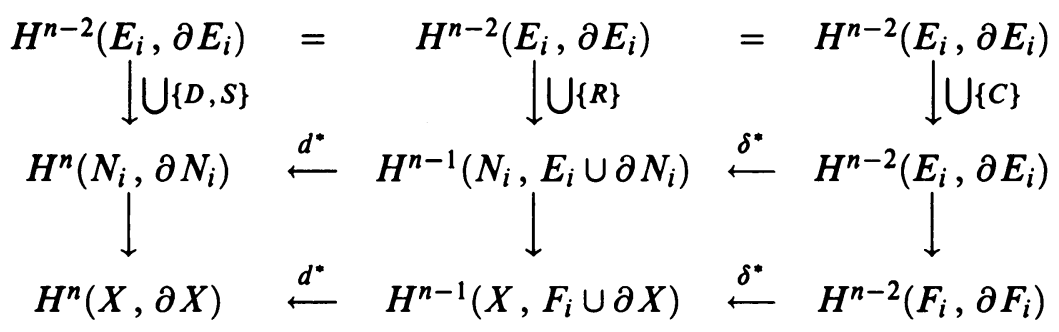

where we identify $D$ with a fiber of $N_{i}$.

In this diagram we use $\{C\}$ to denote the unique class of $H^{0}\left(E_{i}\right)$ restricting to the class $\{C\} \in H^{0}\left(D_{i}\right)$, where $D_{i}$ is the fiber of $N_{i}$ over any point of $\operatorname{Int}\left(E_{i}\right)$. This class is obviously just the nonzero element of $H^{0}\left(E_{i}\right)$. Similarly, 
we use $\{D, S\}$ to denote the unique class in $H^{2}\left(N_{i}, T_{i}\right)$ restricting to the class $\{D, S\} \in H^{2}\left(D_{i}, S_{i}\right)$, with $D_{i}$ as above and $S_{i}=\partial D_{i}$. This class is simply the Thom class of the bundle $N_{i}$ [B, II.2.3]. The vertical map in the upper lefthand corner is actually the composition of the isomorphism $H^{n-2}\left(E_{i}, \partial E_{i}\right) \cong$ $H^{n-2}\left(N_{i}, N_{i} \mid \partial E_{i}\right)$ with the cup product with the class $\{D, S\}$ under the cup product map $H^{n-2}\left(N_{i}, N_{i} \mid \partial E_{i}\right) \times H^{2}\left(N_{i}, T_{i}\right) \rightarrow H^{n}\left(N_{i}, \partial N_{i}\right)$. Also, we use $\{R\}$ to denote the class in $H^{1}\left(N_{i}, E_{i} \cup T_{i}\right)$ which restricts to the class $\{R\}$ in $H^{1}\left(D_{i}, C_{i} \cup S_{i}\right)$, where $C_{i}$ is the center of $D_{i}$, in each fiber, and the middle vertical map is a similar composition.

First, we observe that this diagram commutes. For the top half, this follows directly from $(* *)$, and for the bottom half, this is clear.

Second, we observe that all four vertical maps on the outside of the diagram are isomorphisms. For the map in the upper left-hand corner, this is the Thom isomorphism [B, II.2.3], and for the other three maps, this is clear.

Thus we see that the composition $d^{*} \delta^{*}$ on the bottom line is an isomorphism, and since $\left\{F_{i}, \partial F_{i}\right\}$ (resp. $\left.\{X, \partial X\}\right)$ is the only nontrivial element of $H^{n-2}\left(F_{i}, \partial F_{i}\right)$ (resp. $\left.H^{n}(X, \partial X)\right)$, we have $d^{*} \delta^{*}\left(\left\{F_{i}, \partial F_{i}\right\}\right)=\{X, \partial X\}$, $i=1, \ldots, k$. Then, if $\langle$,$\rangle denotes the Kronecker product of cohomology and$ homology,

$$
\begin{aligned}
1 & =\langle\{X, \partial X\},[X, \partial X]\rangle=\left\langle d^{*} \delta^{*}\left(\left\{F_{i}, \partial F_{i}\right\}\right),[X, \partial X]\right\rangle \\
& =\left\langle\left\{F_{i}, \partial F_{i}\right\}, \partial_{*} d_{*}([X, \partial X])\right\rangle
\end{aligned}
$$

for $i=1, \ldots, k$, and hence $\partial_{*} d_{*}([X, \partial X])=\left[F_{1}, \partial F_{1}\right]+\cdots+\left[F_{k}, \partial F_{k}\right]$ as claimed.

Remark. Note that in the proof of Theorem 2 we removed a neighborhood of $F_{i} \cap\left(F_{1} \cup \cdots \cup F_{i-1} \cup F_{i+1} \cup \cdots \cup F_{k}\right)$. Thus, while we assume $F_{i}$ and $F_{j}$ transverse for $i \neq j$, we need no assumption on triple (or higher) intersections. Similarly, while we assume $F_{i}$ transverse to $\partial X$ for each $i$, we need no assumption on double (or higher) intersections with $\partial X$. (Of course, $\tilde{X}$ will be a manifold if and only if $F_{i} \cap F_{j}=\varnothing$ for $i \neq j$.)

Let us indicate the use of Theorem 2 in conjunction with Theorem 1. For simplicity let us suppose that $\partial X=\varnothing$. Of course, $\pi_{*}([\tilde{X}])=0$, so $[X] \notin$ $\operatorname{Im}\left(\pi_{*}\right)$. However, consider a class $y \in H_{q}(X)$ and the problem of deciding whether $y \in \operatorname{Im}\left(\pi_{*}\right)$ or, equivalently whether $d_{*}(y)=0$. Suppose that $y$ is represented by an embedded submanifold $Y$, i.e., $y=i_{*}([Y])$, where $i: Y \rightarrow$ $X$ is the inclusion. Of course, if $Y \subset F$, then clearly $y \in \operatorname{Im}\left(\pi_{*}\right)$. Otherwise, we may suppose that for some $j \leq k, Y$ intersects $F_{1}, \ldots, F_{j}$ transversely and $F_{j+1}, \ldots, F_{k}$ not at all and that $G_{i}=F_{i} \cap Y, i=1, \ldots, j$, are pairwise mutually transverse in $Y$. Let $G=F \cap Y=G_{1} \cup \cdots \cup G_{j}$. Then we have a commutative diagram

and

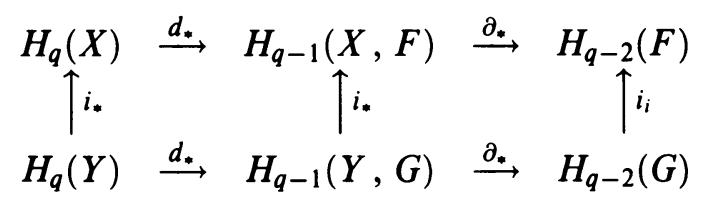

$$
\begin{aligned}
\partial_{*} d_{*}(y) & =\partial_{*} d_{*}\left(i_{*}([Y])\right)=i_{*}\left(\partial_{*} d_{*}([Y])\right) \\
& =i_{*}\left(\left[G_{1}\right]+\cdots+\left[G_{j}\right]\right)=g \in H_{q-2}(F) .
\end{aligned}
$$


Thus, if $g \neq 0$ in $H_{q-2}(F)$, then $d_{*}(y) \neq 0$ and $y \notin \operatorname{Im}\left(\pi_{*}\right)$. On the other hand, if $g=0$ and $H_{q-1}(X)=0$, then $d_{*}(y)=0$ and $y \in \operatorname{Im}\left(\pi_{*}\right)$.

\section{REFERENCES}

[B] W. Browder, Surgery on simply connected manifolds, Ergeb. Math. Grenzgeb. (3), vol. 65, Springer-Verlag, Berlin, Heidelberg, and New York, 1972.

[LW1] R. Lee and S. H. Weintraub, The Siegel modular variety of degree two and level four: a report, Arithmetic of Complex Manifolds (W.-P. Barth and H. Lange, eds.), Lecture Notes in Math., vol. 1399, Springer-Verlag, Berlin, Heidelberg, and New York, 1989.

[LW2] _ The Siegel modular variety of degree two and level four (to appear).

(R. Lee) Department of Mathematics, Yale University, New Haven, Connecticut 06520

(S. H. Weintraub) Department of Mathematics, Louisiana State University, Baton ROUGE, LOUISIANA 70803-4918 